\title{
Flow conveying and diagnosis with carbon nanotube arrays
}

\author{
Jijie J Zhou ${ }^{1,3}$, Flavio Noca ${ }^{2}$ and Morteza Gharib ${ }^{2}$ \\ ${ }^{1}$ Bioengineering, California Institute of Technology, 1200 E California Blvd., Pasadena, \\ CA 91125 , USA \\ ${ }^{2}$ Aeronautics and Bioengineering, California Institute of Technology, 1200 E California Blvd., \\ Pasadena, CA 91125, USA \\ E-mail: jijie@alumni.caltech.edu
}

Received 1 June 2006, in final form 18 July 2006

Published 7 September 2006

Online at stacks.iop.org/Nano/17/4845

\begin{abstract}
Dense arrays of aligned carbon nanotubes are designed into strips, nanowicks, as a miniature wicking element for liquid delivery and potential microfluidic chemical analysis devices. Liquid wicks away along the nanowicks spontaneously. This delivery function of nanowicks enables novel fluid transport devices to run without any power input, moving parts or external pump. Flow around the opaque nanotubes can be detected either directly or indirectly. Direct signals of the flow come out of dyed liquid or from the liquid-air interface; indirect signals are detected through observing surfacetension-induced deformation and dislocation of the nanotubes. Here we show that flow progression around and inside nanowicks is sensitive to liquid properties. Different flow progression leaves different traces of liquid. These traces not only allow liquid diagnosis any time after sampling, but also enable analysis of flow at a nanoscale resolution with scanning electron microscopy.
\end{abstract}

(Some figures in this article are in colour only in the electronic version)

\section{Introduction}

Submicron and nanometre scale pores have been used for molecular sieving, as in electrophoresis and chromatography. Placing such mesopores into microfluidic devices can facilitate quick turnover, cost-effective, and point-of-use diagnostics. Most microfluidic devices currently rely on large auxiliary setups and external power input to drive liquid through channels [1-4]. Flow through nanoscale interstices for lab-ona-chip applications has been less frequently employed, because the required pressure gradient to push liquids through small channels and small interstices becomes unreasonably large, or in other words, the required pressure gradient increases inverse-proportionally to the fourth power of the characteristic size [5]. There are growing needs for novel micromechanical system designs. We have designed an on-chip element for conveying nanoporous flow with intrinsic interfacial forces.

Dense arrays of aligned carbon nanotubes (CNTs) have been used as field emission sources [6], as electrochemical

\footnotetext{
3 Author to whom any correspondence should be addressed. Present address: School of Materials Science and Engineering, 50 Nanyang Avenue, 639798
} Singapore. electrodes [7], and in CNT-polymer composite [8]. Fabrication of surface patterns of CNT arrays has become mature over the past five years [7, 9]. This work takes advantage of such fabrication - it combines top-down lithography and bottom-up self-assembly approaches in micro/nanotechnology to make a low cost, high throughput chip device that relies on nanometre-scale dimensions to attain its functions. We use a strip, comprising dense carbon nanotubes, to guide small amounts of liquid flow with wetting and spreading forces. This spontaneous liquid-delivery process is an analogue to 'wicking' in chromatography. Consequently, we call a strip full of dense arrays of nanotubes for liquid delivery a nanowick.

Wicking is determined by interfacial tensions and hydrodynamics $[10,11]$. The contact angle of a single carbon nanotube has been theorized to increase with the decreasing radius of the tube [12]. The equilibrium contact angle for nanotubes $20 \mathrm{~nm}$ in diameter (the typical value of most nanotube carpet samples used in this research) should be about $83^{\circ}$ theoretically [12], or $80.1 \pm 3.6^{\circ}$ experimentally [13]. However, once the nanotubes assemble into a carpet of aligned nanotube arrays, the carpet, as a whole, functions as a superhydrophobic (contact angle higher than $150^{\circ}$ ) surface [14]. The 
hydrodynamics of pure water passing CNT arrays have been studied numerically for forced imbibition, and drag coefficients are found in reasonable agreement with the macroscopic, Stokes-Oseen, solution [15]. Surfactants are employed for aqueous imbibition in this work to overcome the transversal hydrophobicity of the nanotubes and the super-hydrophobicity atop. The addition of surfactants is widely used to accelerate the capillary penetration of aqueous solution in hydrophobic porous bodies. Such acceleration of capillary impregnation by the addition of a surfactant incorporates a diffusion mechanism of the surfactant into the wicking system [16]. In a conventional hydrophobic capillary, the advancing speed of the liquid front is limited by the diffusion rate of the surfactant. Surfactant molecules have to diffuse in advance of the wetting front to enable liquid to impregnate [16]. Surfactants adsorb strongly to graphite [18-20]. Adsorption of surfactants onto carbon nanotubes enhances a concentration gradient in the advancing front by limited diffusion. We have discovered that this concentration gradient spontaneously drives an interstitial flow passing the dense carbon nanotubes, as in the Marangoni [21] effect. Because of this Marangoni effect, almost pure water advances at the front in the nanotube arrays. That pure water leads the front of surfactant solution has been observed in hydrophilic pores that are smaller than a critical value, due to the limited diffusion rate of surfactant molecules [17].

Spontaneous liquid flow along nanowicks is guided by interfacial forces. This delivery function of nanowicks enables novel fluid transport devices to run without any power input, moving parts or external pump. Besides liquid delivery, the nanotubes themselves can be treated by chemicals in the solution during wicking. Because the fibrous structure of nanowicks provides a large surface-to-volume ratio, the nanowicks can be designed to carry catalysts and reactive agents, or utilized as sieving matrices for molecular separations according to size or morphology, which makes nanowicks a good candidate for potential microfluidic sampling and chemical analysis devices.

\section{Experimental methods}

There are two typical approaches to build a nanoscale analytic device or system-top-down and bottom-up. Topdown techniques create micro/nanoscale mechanical structures out of bulky materials. But device dimensions by topdown techniques are limited by the resolution of electronbeam (e-beam) lithography, etch roughness, and other factors. Bottom-up techniques chemically synthesize nanostructured materials such as carbon nanotubes. The achieved structure can be smaller and smoother than one created with e-beam lithography. Large-scale integration of nanoscale functional structures with top-down fabrication techniques becomes a crucial challenge. On the other hand, bottom-up techniques only create materials, not devices. We combine these two approaches to make cheap, high throughput devices that rely on nanometre-scale dimensions to attain their functions.

\subsection{Fabrication}

A nanowick fabrication involves two major steps: (1) patterning the catalyst, and (2) growing CNTs [7, 9]. Because CNTs only grow from where catalyst exists, patterns, in which

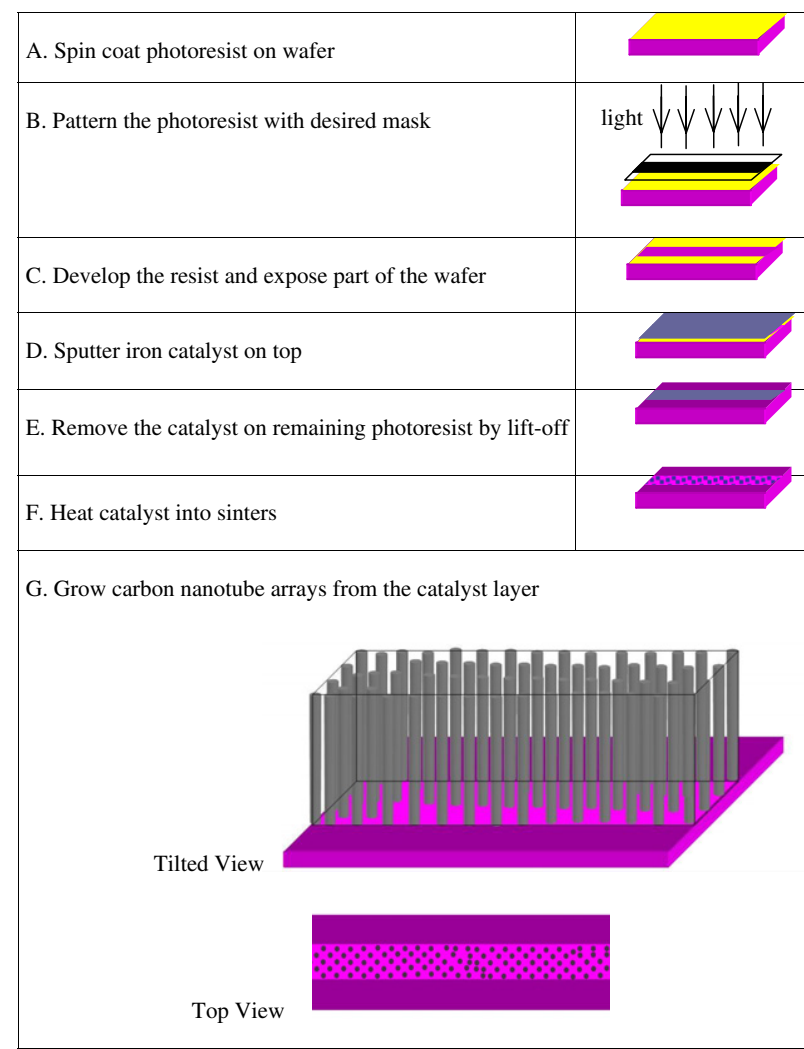

Figure 1. A diagram of nanowick fabrication and its final configuration. A-G: patterns in the catalyst layer are fabricated with photolithography. F, G: nanotubes are produced in arrays with individual nanotubes being vertically oriented with respect to the substrate. The nanotubes only exist in the designed regions.

CNTs exist on a substrate, are determined by arrangement in their catalyst layer. Therefore, scaled-up functional devices with nanoscale structures can be obtained without post-growth manipulation on the nanotubes. Furthermore, if desired, the CNTs in such patterned strips are ready for encapsulation or chemical treatment. Arbitrary design of the catalyst deposition layer by photolithography allows any desired surface pattern of nanotube mats to be grown, and their location on the wafer to be precisely controlled. Depending on the application, the patterns of carbon nanotube mats themselves may be preferred rather than their particular location on the wafer. In such cases, patterns can even be generated without lithography [22, 23].

Patterned catalyst layers can be obtained in many ways [7]. One way, involving photolithography, is illustrated in figure 1 as fabrication steps. A $6-8 \mathrm{~nm}$ thin iron (Fe) film is coated onto micropatterned resist in a magnetron sputter. Remaining resist is removed with acetone, lifting off the iron film atop. Consequently, there remains a patterned iron film on the substrate. Because of the temperature requirement during growth, quartz and thermal oxidized silicon wafers are chosen as the substrate for this work.

Carbon nanotubes are grown with a modified recipe emulated from Hongjie Dai group's 1999 work at Stanford University [24, 25]. Nanotubes are typically grown at high temperature $\left(>600^{\circ} \mathrm{C}\right)$ with a steady supply of carboncontaining gas over a substrate that is adequately layered, in some regions, with a thin coat of catalyst. The nanotubes 

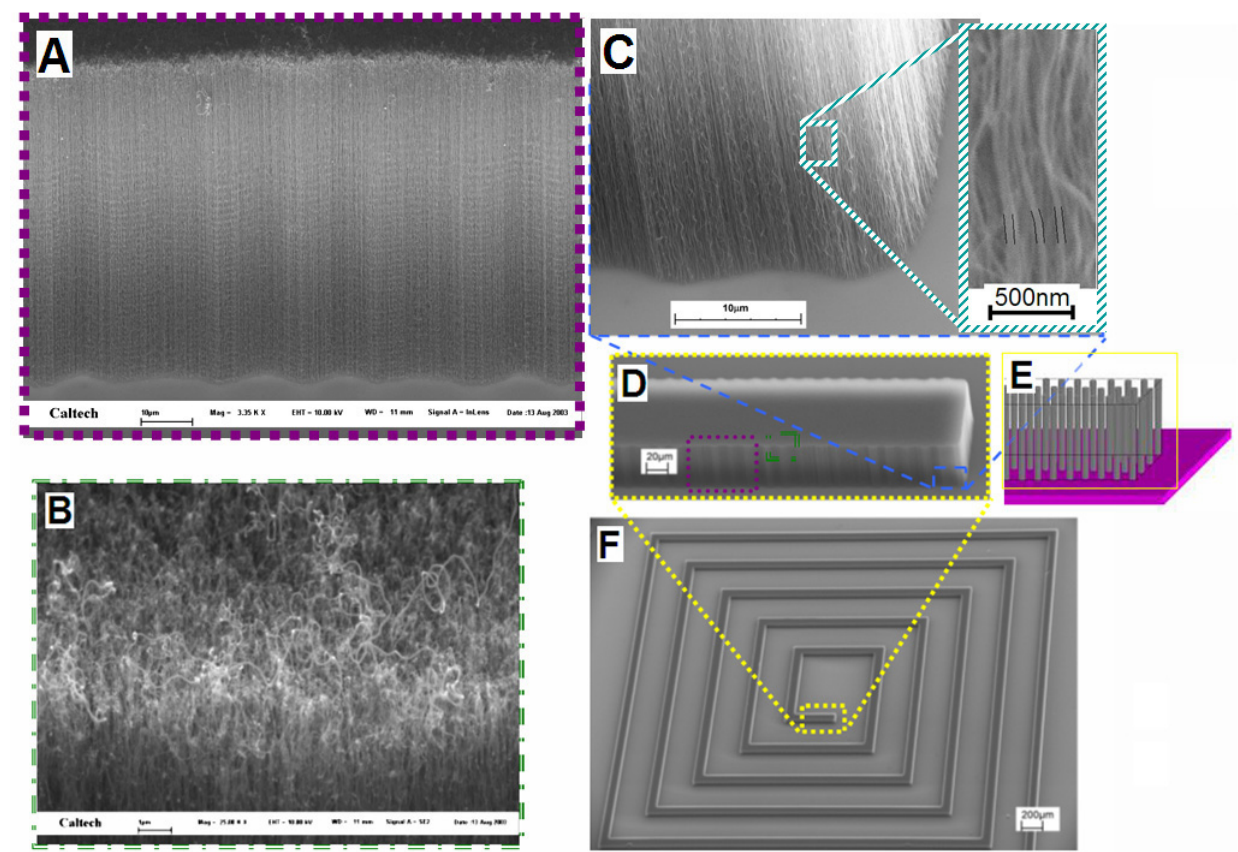

Figure 2. Hierarchical structure of a nanowick in $45^{\circ}$ tilted views. (A) Sidewall of a nanowick; (B) the nanowick top surface; (C) the nanotube roots on the substrate; inset: $20 \mathrm{~nm}$ thick carbon nanotubes as the building block for the nanowick; three nanotubes are sketched out to compare with the deeper ones behind them (note: marginal nanotubes look thicker than inside tubes because of the charging effect by the electron beam); (D) a segment of a $60 \mu \mathrm{m}$ high $100 \mu \mathrm{m}$ wide nanowick, comprising the nanotubes; (E) diagram of the structure in (D); (F) a nanowick with arbitrary micro-patterns can be centimetres long.

can be produced in large, well-ordered arrays with individual nanotubes being vertically oriented with respect to the substrate, due to the crowding effect and van der Waals interaction [7]. Such nanotube/nanowick samples are stored in dry cabinets and desiccators. No shelf-life issue has been encountered so far.

Nanowicks are grown on flat wafers, consisting of nanotubes vertical to the wafer. The overall dimension of a nanowick device is of the order of centimetres; the delivery element is often designed to be tens to hundreds of microns in width and height for easy optical observation and flow diagnosis. The building block-nanotubes-is approximately $20 \mathrm{~nm}$ thick and tens to hundreds of microns high with a centre-to-centre space being on average $60 \mathrm{~nm}$. (The edgeto-edge spacing is on average $40 \mathrm{~nm}$. The value is calculated from nanotube site density.) This hierarchical structure is demonstrated with a photo-lithographed nanowick in figure 2 , where $45^{\circ}$ tilted views of a $100 \mu \mathrm{m}$ wide $60 \mu \mathrm{m}$ high nanowick are given at different magnifications. A segment of curb-like structure, standing on top of a horizontal wafer as shown in figure 2(D) and sketched in figure 2(E), is part of a centimetre long nanowick (figure $2(\mathrm{~F})$ ), consisting of densepacked vertical carbon nanotubes (figure $2(\mathrm{C})$ ). The nanotubes are roughly $20 \mathrm{~nm}$ thick, and better revealed in close-up views: figure 2(A) shows the side wall of a nanowick and each upright line represents a carbon nanotube; figure 2(B) gives a tilted view of the nanowick top. In the near top region, the nanotubes grow to be less aligned, as the crowding effect becomes less.

\subsection{Flow detection method}

The dense opaque nanotubes on a clear wafer enable us to distinguish inside imbibition flow from superficial spreading.
In contrast, traditional translucent porous material confuses the liquid location. Interstitial flow is visualized through clear (quartz) wafers in an inverted fluorescent microscope with the addition of dye molecules in the solution. Because visible light cannot penetrate the dense arrays of carbon nanotubes, the fluorescence signal is stimulated and received from the clear wafer side. Consequently, the signal is inside the nanotube arrays/nanowicks and not superficial. Since we use an inverted fluorescence microscope, the view through the substrate wafer side is named the bottom view. None of the surface flow on the nanotube free-end side is visible from the bottom view. The liquid motion on the nanotube free-end side, surface flow, is observed from the top with upright long-working-distance microscopes. Dye addition is not required for the top surface flow observation. Video signals from the microscope CCD (29.97 frames per second) are recorded by a computer (high resolution) and camcorder (long-term low-resolution storage). Visible displacement from frame to frame is then measured in software such as Adobe Photoshop.

Surface forces, such as liquid-air surface tension, on minute structures are so distinct that any liquid will always leave a trace in delicate nanostructures. Scanning electron microscopy (SEM) is employed for high-resolution dry morphology studies of nanowicks before and after liquid tests. The consequential configuration changes of the nanotube arrays can be detected dynamically under optical microscopy. In this case, the action of surface tension can help visualize part of the interstitial front by shrinking of the sidewalls, where liquid existing on one side pulls them in. Detection of the front with surface tension under optical microscopes has a resolution better than a few microns. 
The surface tension effect, and its alteration in the nanoarray configuration, can be confirmed in SEM in great detail after the liquid dries up. Three types of action by surface tension are seen in the nanowicks:

(i) Shrinking of the nanowick sidewalls as the interstitial flow advances. This can be observed dynamically in optical microscopy when nanotubes are tens of nanometres long.

(ii) Forming of trenches/cracks on liquid-air borders inside nanowicks as the liquid dries. This happens after the liquid stops advancing, as shown in figure 7(A).

(iii) Opening of circular, polygonal, or slender cells everywhere in the wetted nanotube arrays during dewetting [26]. The cell structure, void in nanotube arrays, can be used as markers showing the regions where liquid has reached (figure 7(B)).

These traces not only enable high-resolution morphological analysis of the liquid front, but also allow liquid diagnosis anytime after sampling because the new configuration in nanoarrays is robust and remains. Therefore, the liquid trace can be utilized to decipher flow without direct observation of the flow itself. The match of flow with its alteration in the nanotube forest is illustrated in figure 7(A).

During chemical functionalization of nanowicks, one can stay away from the liquid-air surface tension by critical point drying [27] in order to preserve the aligned structure of the nanotubes.

\subsection{Supply droplet}

As a prototype, we adopt an easy input interface by placing a small drop of solution $(0.5-2.5 \mu \mathrm{l})$ beside exposed nanowicks, or on an inlet auxiliary pad, as illustrated in figure 3 . The volume is measured with a Hamilton syringe, which is held in place with a micromanipulator. The droplet is suspended from a Teflon tip of the syringe before it is moved down and contacts the nanowick. The droplet is assumed stationary with no injecting speed. The droplet spreads upon the hydrophilic substrate wafer. But an outward rivulet along the contacting nanowick decreases the droplet volume, and liquid in the droplet ends up retreating as shown in figure 3(A) inset. The retreat of the triple line may be continuous throughout the drying process, or the rapid depletion of liquid close to the nanowick may create a dry curve inside the droplet and end up draining only the liquid close to the nanowick. All observations of flow progression on nanowicks are based on such a nonconstant inlet condition.

In order to convey water-based solutions through well-known hydrophobic carbon nanotube arrays, we take advantage of surfactants, and use the anionic surfactant SDS (well below critical micelle concentration $(\mathrm{cmc})$ ) or non-ionic surfactant Triton X-100 biological compatible (0.01-0.1 wt\%) aqueous solutions. The addition of surfactants not only accelerates the penetration of aqueous solution in hydrophobic porous structures, but also decreases the rate of evaporation on the free surfaces.

\section{Results and discussion}

The nanowick structure is different from conventional porous fibres in the sense of both configuration and dimension.

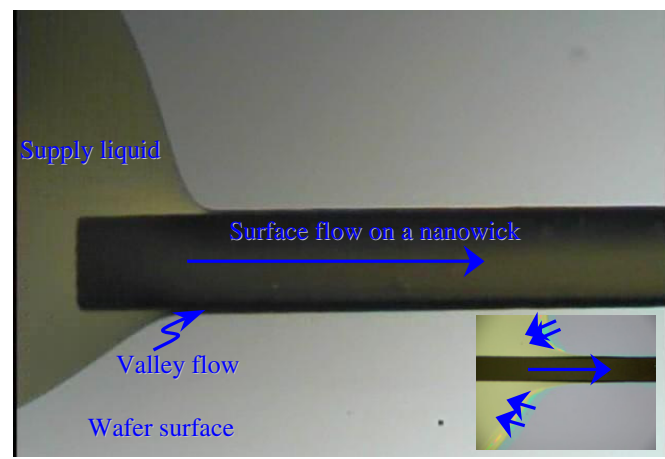

A: Top view of flow on a $3 \mu \mathrm{m}$-high $100 \mu \mathrm{m}$-wide nanowick

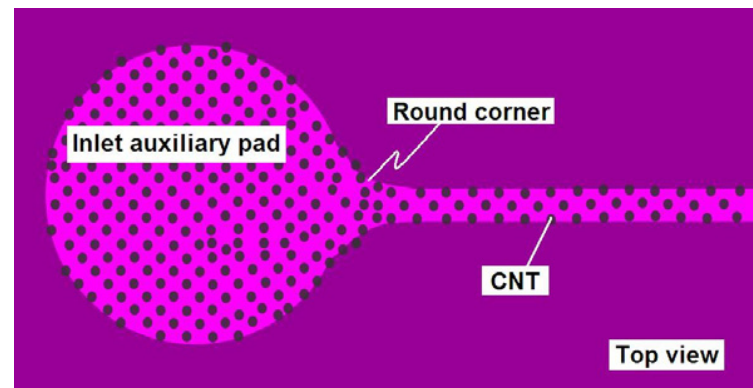

B: Diagram for an inlet auxiliary pad for high aspect ratio nanowicks.

Figure 3. Liquid input for nanowicks. In (A), a liquid droplet is placed at one end of the black strip - top view of a nanowick, and flow is guided by the nanowick. In (B), liquid is placed on a large inlet auxiliary pad in order to prevent liquid from contacting the valley between nanowick sidewalls and substrate (not drawn proportionally). Each black dot represents a top view of an individual carbon nanotube (CNT). The light region is to highlight where the nanotubes exist.

Textile, painting and printing industries have been studying capillary flow on microscale porous fibres or layers. However, liquid flow through nanoscale porous media or fibres has been less frequently studied experimentally [10, 17]. The nanowicks in this work are 30-1700 $\mu \mathrm{m}$ wide, 3-80 $\mu \mathrm{m}$ high, and up to $2 \mathrm{~cm}$ long before turning. (Refer to section 2.1.) Considering the nanowick as a strip of porous media, its pores are anisometric, and the transverse pore size is three orders of magnitude smaller than that of conventional porous media. In addition, the solid substrate that supports the nanowicks brings in a V-shaped groove, formed between a sidewall of the nanowick and supporting substrate.

As the overall structure shrinks, free surface flows outside the porous body become distinct. Flows have been observed in three feature topographies relative to nanowicks:

(i) between an edge of a nanowick and the supporting wafer-valley flow;

(ii) over the top roughness of nanotube arrays-top surface flow;

(iii) through the interstices of dense nanotube matsinterstitial flow.

The overall progression of liquid flow around and inside a nanowick is sensitive to nanowick configuration, liquid properties, inlet condition and operating ambients. A diagram of flow progression is exemplified in figure $6(\mathrm{C})$. Interstitial 
flow, from this work's observation, is slower, but more persistent than the other two, because nanopores produce both the largest viscous drag and the largest wetting force in flow. The former presents as 'slower'; the latter presents as 'persistency'.

\subsection{Valley flow and its control}

When nanowicks protrude out of supporting substrate like a cliff, we name the flow along, but outside, nanowicks on the support substrate a valley flow. Valley flow also refers to flow in between two nanowicks when they are close to each other, because flow along an open channel with a rectangular crosssection shares the same principles of flow along V-shaped grooves beside nanowicks.

A valley is the same as a V-shaped groove surface with an opening angle of $90^{\circ}$, especially for films thinner than the capillary length. The capillary length in our experiments is of the order of a few millimetres, greater than the nanowick height. Valley flow can develop without top free-surface flow or interstitial flow when a nanowick is replaced with a solid wall. Therefore the valley flow is induced by the valley's shape alone, the same as V-groove flow [28-30]. The cross-sectional profile for valley flow, when noncontacting with the top surface flow, should be concave [30]. When the supply droplet is higher than the nanowick, the valley flow receives part of its liquid supply from the top surface flow on approximately the first millimetres along the rivulet. The cross-sectional profile for surface flow in the absence of valley flow and interstitial flow should be parabolic [31]. When surface flow is in direct contact with valley flow, valley flow gives in and adapts a convex shape to live in harmony with the positive pressure in the supply droplet and the surface flow. The cross-sectional profile of valley flow is only observed indirectly through light interference patterns. In addition, valley flow on the first millimetres appears narrower than the flow of about tens of millimetres long that developed after the first few millimetres.

If valley flow is not desired for integration of nanowicks, it can be minimized, and even prevented by proper design of the inlet or nanowick surrounding configuration. Valley flow is limited by the side wall height and the capillary length. Since valley flow is induced by the tilted V-shape groove and its corresponding capillary pressure, it can be avoided by separating the $\mathrm{V}$-groove and flow, or by providing a negative Laplace pressure that contradicts the capillary pressure at the valley.

The following is a list of minimization and prevention methods for valley flow:

(i) Use a low height-to-width aspect ratio nanowick, as in figure 3 .

(ii) Induce concave curvature in the supply droplet, as in figure 4(B).

(iii) Prevent upstream liquid at the inlet from contacting the groove $[2,32]$.

(a) By growing nanowicks in a valley and covering the sidewall.

(b) By building dams for the valley flow.

(c) By filling in the valley with encapsulation materials.

(d) By inputting flow away from nanowick edges on an inlet auxiliary pad.
The inlet auxiliary pad is made of the same nanotube arrays and is in contact with a strip-shaped nanowick as in figure 3(B).

Concave corners of nanowicks induce capillary flow. In order to eliminate liquid loss into concave corners, circuits of nanowicks require rounding of the concave corners on the liquid transport pathway [33], in contrast to the counterpart chip design in the semiconductor industry.

\subsection{Free-surface flow atop nanowicks}

Liquid placed on top of a nanowick will first flow through the asperities of the tip surface, similar to capillary spreading along hydrophilic microstripes [31]. Such free-surface flow on the asperities is topography-driven (roughness-assisted) spreading [34-36]. The spiky top surface, consisting of nanotube tips, provides nanoscale roughness. Surface roughness can exaggerate a material's natural wettability. That is, roughness improves wetting and spreading on hydrophilic surfaces, and magnifies the hydrophobicity for hydrophobic ones [36, 37].

On a low aspect ratio nanowick (where the height of a nanowick is much smaller than its width), top surface flow dominates valley flow, if the latter exists, and the valley flow becomes less important than the surface flow in terms of the volume flow rate. The valley flow beside a $3 \mu \mathrm{m}$ high $100 \mu \mathrm{m}$ wide nanowick is less than $4 \mu \mathrm{m}$ thick ( $4 \mu \mathrm{m} \ll 100 \mu \mathrm{m})$, as exemplified in figure 3(A). That beside a $40 \mu \mathrm{m}$ high $1.8 \mathrm{~mm}$ wide nanowick is approximately $15 \mu \mathrm{m}$ thick $(15 \mu \mathrm{m} \ll$ $1.8 \mathrm{~mm})$

\subsection{Transition to interstitial dominant flow}

In reality, liquid first spreads on a composite air-solid surface [38]. Consequently, surface flow creates air pockets inside the nanowicks, and trapped air generates a resistance to liquid penetration along a nanotube's long axis. As the front of surface flow moves farther away from the supply droplet, the surface flow slows down because the capillary pressure drop has to work over a larger distance. Lateral interstitial flow then takes over and leads the surface flow. Figure 6(C) gives a schematic diagram of flow progression, neglecting the valley flow.

When the surface flow slows down gradually to a speed of the same order as that of vertical penetration, air inside the nanowick has enough time to be displaced. Consequently, there are no trapped bubbles at this stage. This stage is a transition from surface dominant flow to interstitial dominant flow. In this transition, the liquid interface in the nanotube matrix is inclined, and is seen as a cloud, out of the focal plane, ahead of the focal fluorescence border (figure 4(BC2)). Please note that no top surface flow is seen due to the opaqueness of the nanotubes. This cloud becomes easier to interpret when compared with the colour gradient in figures 4(C1), (C3) and (BC3), where the liquid edge appears sharp and the border is within the focal plane. The light intensity gradient seen on the sharp-edge front results from the fluorescein concentration gradient. The concentration gradient results from (i) the faster adsorption of fluorescein molecules onto the high surface-tovolume ratio nanotube bed and (ii) the slow diffusion rate in nanopores. Further discussion of the concentration gradient is given in section 3.5. 

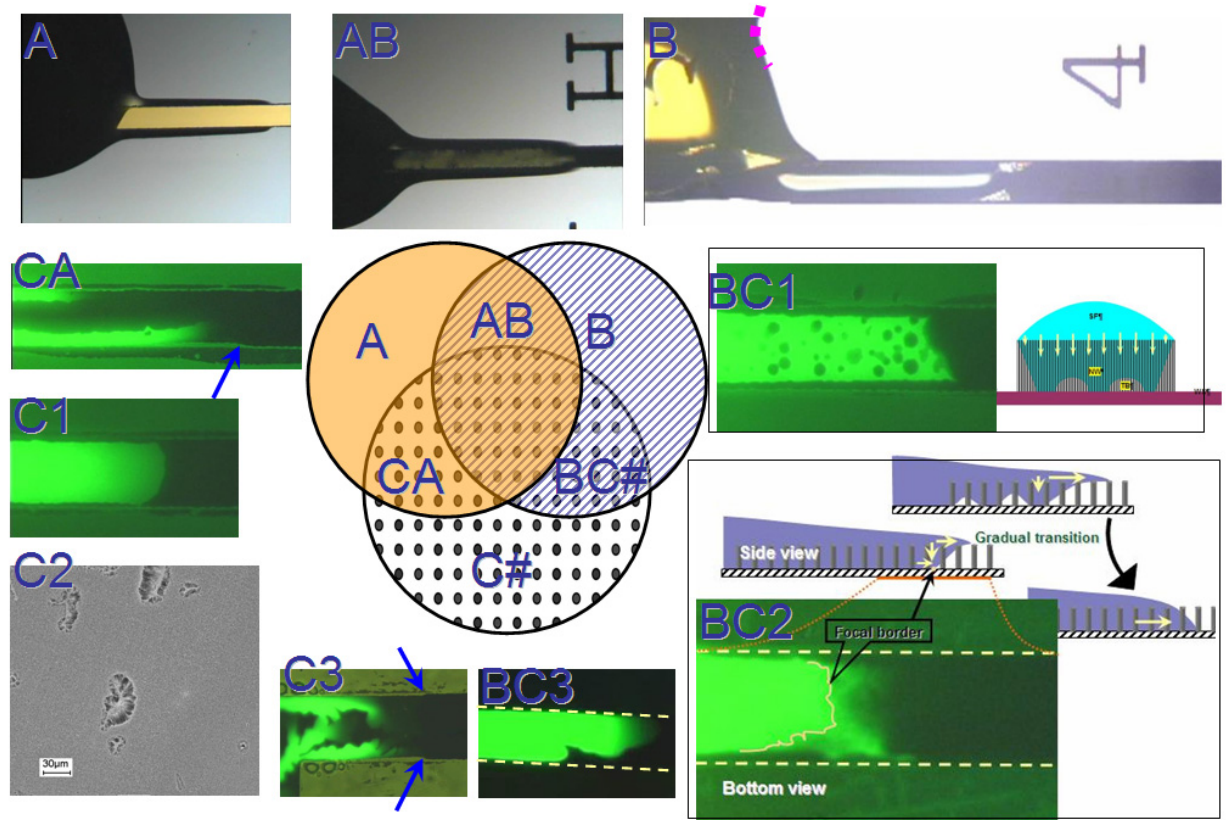

\section{A}

: Valley flow; B

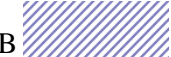

: Top free-surface flow;

$00 \bullet \bullet \bullet$

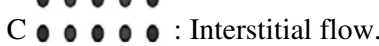

Figure 4. Liquid flow formats around and inside a nanowick. Liquid flows from left to right in all demonstrations. All fluorescein (green) illustrations of flow are viewed from bottom through a clear substrate; others ((A), (AB), (B) and (C2)) are top views from the free-tip side. (A) Valley flow is decoupled from surface flow. It is induced by tilted V-shape grooves as beside a solid step. (AB) Valley flow is supplied, fully or partially, by top surface flow, illustrated by top view on a $40 \mu \mathrm{m}$ high $100 \mu \mathrm{m}$ wide nanowick. (B) Surface flow without valley flow, illustrated by top view on a $40 \mu \mathrm{m}$ high $100 \mu \mathrm{m}$ wide nanowick. (BC1) Liquid seeps into nanowick interstices from the top, where inside air is trapped. Left: bottom view of trapped bubble (the black circular holes inside the fluorescein colour). Right: cross-sectional schematic diagrams of seeping for high nanowicks. (BC2) Top liquid seeps into nanowick interstices, where air finds a vent and is displaced. It is a transition stage as the surface flow gradually slows down. Insets: side view schematic diagrams of the transition. (BC3) Fingers form in surface flow and then invade the nanowicks. (C1) and (C3) Lateral interstitial flow passing nanotubes one by one with the Marangoni effect (refer to the phase diagram in figure 6). (C2) Lateral flow passing nanotubes due to lateral continuity from the substrate (refer to the phase diagram in figure 6). (CA) Edge flow along the side walls and mainly inside nanowicks. Upstream liquid in the valley is taken into nanowick interstices.

\subsection{Typical observations of lateral interstitial flow}

In lateral interstitial flow, liquid is in contact with the bottom wafer when passing nanotube arrays. As a result there is no trapped air or bubbles. Examples are given in figures $4(\mathrm{C} 1)$ and (C3), where the front either displaces like a piston, or branches. Such distinct behaviours result from the difference in surfactant concentration. When the surfactant concentration becomes close to zero, discrete clusters can be seen as in figure $4(\mathrm{C} 2)$ or $7(\mathrm{C})$. These patterns are further discussed in section 3.5 with a phase diagram.

Branching wicking refers to the flow that branches randomly at the front while moving forward inside the nanotube forest. It is observed at stagnant liquid boundaries, when an aqueous droplet with very low surfactant concentration is pinned and barely spreads itself on top of a nanowick. Branching wicking can carry liquid hundreds of microns away. The branching front has been observed with fluorescein in water, $1 / 2 \mathrm{cmc}$ SDS, and $2 / 3 \mathrm{cmc}$ Triton. (Note: fluorescein is a mild surfactant itself, SDS $\mathrm{cmc}$ is $\sim 8.3 \mathrm{mM}$ and Triton cmc is $\sim 0.23 \mathrm{mM}$. The two concentrations were tested without the addition of fluorescein.) The spreading force for low surfactant solution is small, but being close to the supply liquid offers low viscous drag for liquid to extend. Low viscous drag introduces viscous instability and leads to the branching phenomena.

Due to the high viscosity, decrease in surfactant concentration at the front as liquid advances, and flow conditions from top surface flow and valley flow, some interstitial flow has been observed as partial invasion, in that liquid invades part of the nanowick area and leaves the other part untouched. The partial invasion has been seen as fingerlike invasion, or edge flow, shown in figures 4(BC3) and (CA). In finger-like invasion, fingers first form on the surface of nanotube tips and then seep into the interstices. The front of the surfactant solution on the surface may break into fingers due to the instability at the leading edge [39]. Edge flow, seen as interstitial flow, preferentially runs along the edges of a nanowick. The edge flow might employ nanowick sidewalls as a pathway to supply liquid and to lower the viscous drag. This is inferred from the coexistence of bulky liquid in contact with the sidewalls upstream, such as valley flow. But this work is not able to distinguish a thin film on the sidewalls. Even if it does exist, detection with the current setup is still a challenge. Flow formats are summarized in figure 4. 


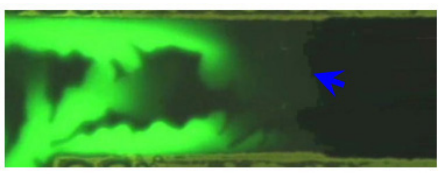

A: Front conformation when it stops moving

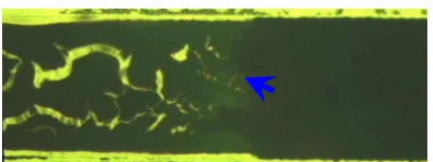

B: Open cracks after solution dries up

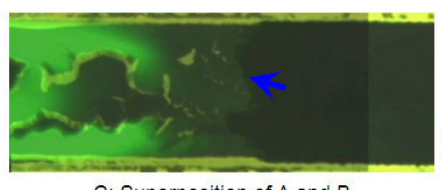

C: Superposition of $A$ and $B$
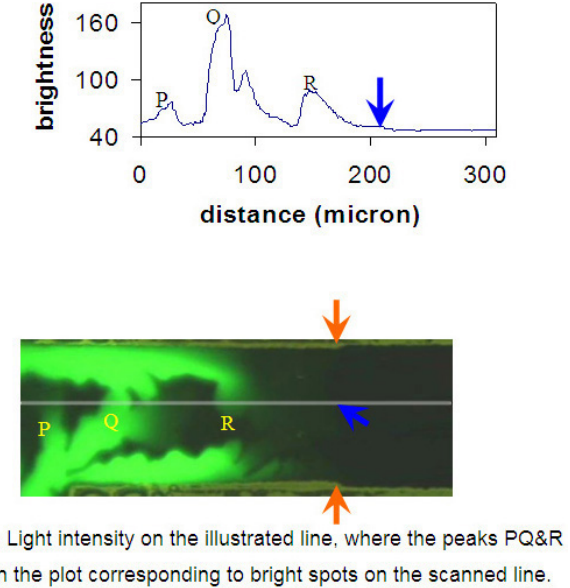

Figure 5. Light intensity change and crack location near an interstitial front. These open cracks (or trenches in high magnification micrographs) are found in the wetted regions without apparent green colour. Blue arrows point at one spot in the nanowick under different views. Orange arrows illustrate the front location observed through sidewall shrinking. The nanotubes are $40 \mu \mathrm{m}$ high; the wick is designed as $100 \mu \mathrm{m}$ wide.

\subsection{Forces involved in interstitial wicking}

Interstitial wicking is the most desirable format in order to take advantage of porous structure of nanowicks for microfluidic chemical analysis. We discover that the Marangoni force drives the spontaneous lateral interstitial flow. The Marangoni effect [21] is another mechanism of droplet spreading, other than restoring balanced surface tension at the three-phase contact line. The spatial variation in surface tension at a liquid/gas surface results in added tangential stresses at the surface; these tangential stresses result in a surface traction that acts on the adjoining fluid, giving rise to the fluid motion in the underlying bulk liquid. Therefore, liquid tends to move in a direction from a low to a high surface tension, and to create Marangoni flow. (Note: the addition of surfactants decreases the surface tension of water.)

Surfactant concentration is not observed directly, but one can infer that a surfactant behaves similarly to fluorescein. The fluorescein concentration varies dramatically near the front, and is seen as a light intensity gradient on the focal plane in figures 4(C1), (4C3), (4BC3) and 7(A). The value of light intensity is plotted as a variation in brightness, shown for one line along the flow direction in figure 5(D). The light intensity change corresponds to concentration changes, not thinning of the liquid layer, because the liquid thickness there should be of the order of microns and more, in order to mechanically deform the nanotubes and open cracks as in figures 5(B) and 7(A). In figure 5 , cracks that form on the wetted region prove that liquid in the nanotube forest is thick enough to cause a green signal, provided that the fluorescein concentration has not decreased. There are very few fluorescein molecules at the front. Therefore, very low surfactant water sees a hydrophobic surface at front. Three-phase contact line disparity is not enough to drive the interstitial flow. A compensatory wetting force is created by the concentration gradient near the front. This gradient results from these combinative effects: (i) fast adsorption on to graphene [18-20], (ii) large surface-to-volume ratio in nanowicks, (iii) slow diffusion in nanopores $[16,17]$ (the surfactant molecules cannot get to the front fast enough). The front position of interstitial flow can be detected with surface tension action. Shrinking occurs as liquid arrives at the sidewall of a nanowick, and the deformation remains after liquid evaporation. An example of interstitial front indicated by the shrinkage at sidewalls is given in figure 5(D), pointed out by orange arrows. These front positions can be confirmed with the brightness profile scanned on a line along flow direction, or cracks and trenches formed along the edges of wetted regions in nanotube arrays.

Experimental observations of flow in this work are on a scale that is much larger than the pore size. The recorded branching fronts are a direct proof of the discrete behaviour of imbibition in pores. Lenormand summarized the competition of wetting forces and viscous drag, in the field of flow inside porous media, into phase diagrams [40]. Studies on the stability and existence of a liquid interface in porous media display the different transport behaviors as qualitative domains in three phase diagrams: one for drainage and two for imbibition [10, 40]. The nanotube arrays for this work have a small pore aspect ratio, $\sim 1.6$. Consequently the lateral interstitial flow in nanotubes is compared with the phase diagram for imbibition in small aspect ratio porous media. For imbibition of small aspect ratio pores, the main mechanism is pore invasion. During invasion of the wetting fluid, the pore throats are filled rapidly, as long as the meniscus from the pore body entirely touches the walls around that throat. Lenormand phase diagrams, without involving the Marangoni effect, employ the capillary number $(C a=V \eta / \gamma)$ and the viscosity ratio $\left(\eta / \eta_{0}\right)$ of the two fluids involved to illustrate the competition of viscous and capillary forces. (Note: $\eta_{0}$ is the viscosity of the defending fluid, air, in this work.) Four typical observations for imbibition in small aspect ratio porous media are: (i) 'capillary branching', (ii) 'stable displacement' of the interface, (iii) disjointed clusters of the imbibing liquid, and (iv) a compact domain with well-defined interfaces that reflect the pore lattice particulars, e.g., a compact wetted domain 


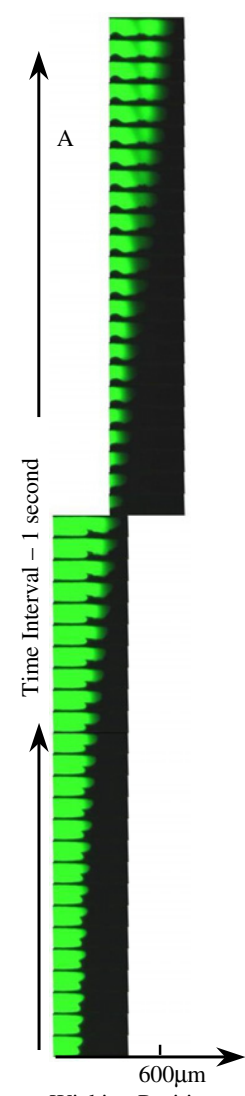

Wicking Position
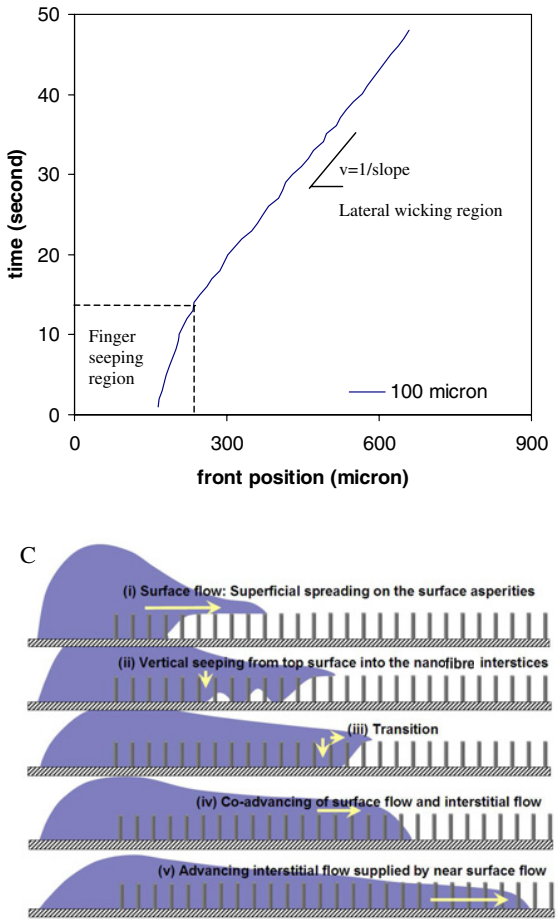

Figure 6. Speed of fingering and lateral interstitial flow. Liquid front displacement on a $100 \mu \mathrm{m}$ nanowick is illustrated by image frames (A) and a plot (B). The plot is extracted from left frames. The lateral wicking can speed up when the Marangoni effect is induced. The lateral wicking shown here has a constant speed $v=12.6 \mu \mathrm{m} \mathrm{s}^{-1}$ for liquid front advancing. (C) A typical progression of flow in nanowicks. The lateral wicking region corresponds to stage (v) in $(\mathrm{C})$.

appears triangular in triangular arrays and square in square arrays.

In the analogous analysis for a phase diagram of nanowick interstitial flow with liquid-air interfaces both on the flow pathway and at the advancing front, the viscous ratio is mapped to viscous effective distance, $l / l_{0}$, where $l_{0}$ needs to be independent of liquid properties, and is chosen as $100 \mu \mathrm{m}$. The viscous effect is reflected by the distance from the imbibing front interface to the bulky supply source. For example, all observed branching fronts are close to the surface flow, such that the viscous effective distance is small. The capillary number in this phase diagram is replaced by effects from both wetting forces - capillary force and Marangoni force, say, $\left(\frac{V \eta}{\gamma}+\frac{\tau \cdot l}{\gamma}\right)$. The augend is a capillary number and the addend is so defined to correspond to the Marangoni effect, where $\tau$ is the Marangoni outward stress and $l$ is the effective distance that $\tau$ acts on. Such a phase diagram for the interstitial flow in nanowicks is illustrated in figure 7. Three behaviours, (i) 'capillary branching', (ii) 'stable displacement', and (iii) disjointed clusters, have been observed as analogues in nanowicking as exemplified in the figure. In the third case-disjointed wetted regions-liquid may flow in a film along the hydrophilic supporting wafer, and aggregate at

$\log \left(\frac{V \eta}{\gamma}+\frac{\tau \cdot l}{\gamma}\right)$

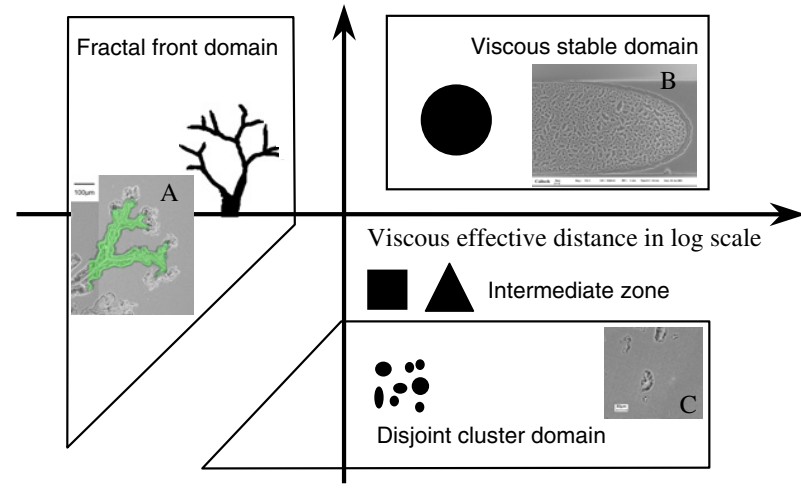

Figure 7. Distribution of qualitative competitive domains for viscous, surface tension and wetting effects, illustrated with SEM images of liquid trace. Nanotubes cover the whole field of view of (A) and (C), the green colour in (A) corresponds to liquid viewed through fluorescence microscopy and grey cracks are viewed from scanning electron microscopy.

random locations to form small isolated wetting regions that are discontinuous from each other. In the case of small pore aspect ratio, high surface tension keeps liquid in the cluster. The fourth case (iv) — that flow sees pore lattice details — is not observed, because our crowding nanotube arrays may not be regular enough to present the phenomena.

This work does not address the issues associated with temperature change nor accompanying changes in physical parameters. Evaporation may lower the temperature by $3{ }^{\circ} \mathrm{C}$ in a layer of thickness about $500 \mu \mathrm{m}$ immediately below the interface [41]. And fluorescein excitation light may increase the local temperature by $4{ }^{\circ} \mathrm{C}$ (measured with a thermocouple). Evaporation affects the late-stage speed, especially, when liquid under observation is heated with excitation light in fluorescence microscopes. This heating effect becomes more distinct, when we encapsulate nanowicks and try to control evaporation. Evaporation condenses the surfactant concentration. This might be responsible for the resuming flow of $0.68 \mathrm{wt} \%$ SDS on $40 \mu \mathrm{m}$ high nanowicks, when valley flow exists. The resuming flow refers to the fact that valley flow and surface flow have been observed to resume themselves from edge flow, and to dash millimetres in the last $20 \mathrm{~s}$ right before all liquid dries up. The resuming flow rapidly pumps liquid into the nanowicks, and its span is $\sim 1 \mathrm{~mm}$, detectable with the naked eye. Systematic investigation on force balance of the resuming flow at late stages has not been done.

\section{Conclusion}

We have introduced a nanowick prototype, consisting of dense arrays of aligned carbon nanotubes, for liquid transport and chemical analysis on microfluidic devices. Interstices among the nanotubes supply durable wicking force by the Marangoni effect. Flow patterns in the interstices are sensitive to surfactant concentrations. Flow progression, depending on inlet 
condition, involves top free-surface flow and possibly valley flow. Valley flow beside nanowicks can be used for fast liquid delivery, and it can be minimized or eliminated, if desired.

The delivery function of nanowicks enables novel fluid transport devices running without any power input, moving parts, or external pump. In addition, the interstitial flow exposes liquid to a large surface-to-volume ratio nanoporous structure. Therefore the delivery and intrinsic nanofibrous nature give nanowicks potential applications in chemical analysis with fluid circuits (micro-chromatography), bodyworn and disposable lab on a bandage, rate control of microcombustion, and microchip cooling circuits (capillary pumping loops).

\section{Acknowledgments}

We would like to thank Michael J Bronikowski for growing the nanotubes for this work in Microdevices Laboratory, Jet Propulsion Laboratory. This work was supported by the NASA Office of Biological and Physical Research (Award No 0452006); and the NSF NER Program (Award CTS-030473).

\section{References}

[1] Stone H A, Stroock A D and Ajdari A 2004 Engineering flows in small devices: microfluidics toward a lab-on-a-chip Annu. Rev. Fluid Mech. 36 381-411

[2] Darhuber A A and Troian S M 2005 Principles of microfluidic actuation by modulation of surface stresses Annu. Rev. Fluid Mech. 37 425-55

[3] Darhuber A A, Valentino J P, Troian S M and Wagner S 2003 Thermocapillary actuation of droplets on chemically patterned surfaces by programmable microheater arrays J. Microelectromech. Syst. $12873-9$

[4] Quake S R and Scherer A 2000 From micro- to nanofabrication with soft materials Science 290 1536-40

[5] Sutera S P and Skalak R 1993 The history of Poiseuille law Annu. Rev. Fluid Mech. 25 1-19

[6] Deheer W A, Chatelain A and Ugarte D A 1995 Carbon nanotube field-emission electron source Science $\mathbf{2 7 0}$ 1179-80

[7] Dai H J 2002 Carbon nanotubes: synthesis, integration, and properties Acc. Chem. Res. 35 1035-44

[8] Jung Y J et al 2006 Aligned carbon nanotube-polymer hybrid architectures for diverse flexible electronic applications Nano Lett. 6 413-8

[9] Hata K et al 2004 Water-assisted highly efficient synthesis of impurity-free single-waited carbon nanotubes Science 306 1362-4

[10] Alava M, Dube M and Rost M 2004 Imbibition in disordered media Adv. Phys. 53 83-175

[11] Alleborn N and Raszillier H 2004 Spreading and sorption of a droplet on a porous substrate Chem. Eng. Sci. 59 2071-88

[12] Neimark A V 1999 Thermodynamic equilibrium and stability of liquid films and droplets on fibers J. Adhes. Sci. Technol. 13 1137-54

[13] Barber A H, Cohen S R and Wagner H D Static and dynamic wetting measurements of single carbon nanotubes 2004 Phys. Rev. Lett. 9282548

[14] Feng L et al 2002 Super-hydrophobic surfaces: from natural to artificial Adv. Mater. 14 1857-60

[15] Walther J H, Werder T, Jaffe R L and Koumoutsakos P 2004 Hydrodynamic properties of carbon nanotubes Phys. Rev. E 69062201

[16] Churaev N V 2000 Liquid and Vapor Flows in Porous Bodies: Surface Phenomena (Moscow: Gordon and Breach)
[17] Starov V M, Zhdanov S A and Velarde M G 2004 Capillary imbibition of surfactant solutions in porous media and thin capillaries: partial wetting case $J$. Colloid Interface Sci. 273 589-95

[18] Patrick H N and Warr G G 2000 Self-assembly structures of nonionic surfactants at graphite-solution inter-faces. 2 . Effect of polydispersity and alkyl chain branching Colloids Surf. A 162 149-57

[19] Patrick H N, Warr G G, Manne S and Aksay I A 1997 Self-assembly structures of nonionic surfactants at graphite/solution interfaces Langmuir 13 4349-56

[20] Richard C, Balavoine F, Schultz P, Ebbesen T W and Mioskowski C 2003 Supramolecular self-assembly of lipid derivatives on carbon nanotubes Science 300 775-8

[21] Marangoni C 1871 Ueber die Ausbreitung der tropfen einer flussigkeit auf der oberflache einer anderen Ann. Phys. Chem. 143337

[22] Noca F, Bronikowski M, Zhou J, Sansom E and Gharib M 2003 Nanowicks: nanofiber-patterned surface for passive fluid transport, nanopumping ultrafiltration, nanomixing and fluid logic US Provisional Patent Patent pending

[23] Zhou J, Sansom E, Gharib M and Noca F 2003 Nanoscale Wicking 56th Annual American Physical Society/Division of Fluid Dynamics (APS/DFD) Mtg (East Rutherford, NJ)

[24] Fan S S et al 1999 Self-oriented regular arrays of carbon nanotubes and their field emission properties Science 283 512-4

[25] Bronikowski M J, Self-assembly process of carbon nanotubes for most samples in this work are done in the JPL Microdevices Laboratory by Michael J Bronikowski

[26] Sansom E, Zhou J, Noca F and Gharib M 2003 Self-assembly patterns formed by lateral capillary force within carbon nanotube arrays 2003 Materials Research Society (MRS) Fall Mtg

[27] Johnston K P and Shah P S 2004 Materials science-Making nanoscale materials with supercritical fluids Science $303482-3$

[28] Mann J A, Romero L, Rye R R and Yost F G 1995 Flow of simple liquids down narrow-V grooves Phys. Rev. E 52 3967-72

[29] Rye R R, Mann J A and Yost F G 1996 The flow of liquids in surface grooves Langmuir 12 555-65

[30] Romero L A and Yost F G 1996 Flow in an open channel capillary J. Fluid Mech. 322 109-29

[31] Darhuber A A, Troian S M and Reisner W W 2001 Dynamics of capillary spreading along hydrophilic microstripes Phys. Rev. E 6403031603

[32] Foster K and Parker G A 1970 Fluidics: Components and Circuits (London: Wiley-Interscience)

[33] Darhuber A A, Troian S M, Miller S M and Wagner S 2000 Morphology of liquid microstructures on chemically patterned surfaces J. Appl. Phys. 87 7768-75

[34] Bico J, Tordeux C and Quere D 2001 Rough wetting Europhys. Lett. 55 214-20

[35] McHale G, Shirtcliffe N J, Aqil S, Perry C C and Newton M I 2004 Topography driven spreading Phys. Rev. Lett. 93036102

[36] Wenzel R N 1949 Surface roughness and contact angle J. Phys. Colloid Chem. 53 1466-7

[37] Onda T, Shibuichi S, Satoh N and Tsujii K 1996 Super-water-repellent fractal surfaces Langmuir 12 2125-7

[38] Seveno D, Ledauphin V, Martic G, Voue M and De Coninck J 2002 Spreading drop dynamics on porous surfaces Langmuir 18 7496-502

[39] Matar O K and Troian S M 1999 The development of transient fingering patterns during the spreading of surfactant coated films Phys. Fluids 11 3232-46

[40] Lenormand R 1990 Liquids in porous-media J. Phys.: Condens. Matter 2 SA79-88

[41] Ward C A and Stanga D 2001 Interfacial conditions during evaporation or condensation of water Phys. Rev. E 64051509 